\title{
Residential Out-of-Home Care Staff Perceptions of Implementing a Trauma-Informed Approach: The Sanctuary Model
}

\author{
Emma Galvin ${ }^{1}$ (D) Renee O'Donnell ${ }^{1} \cdot$ Julie Avery ${ }^{1} \cdot$ Heather Morris $^{1} \cdot$ Aya Mousa $^{2} \cdot$ Nick Halfpenny $^{3} \cdot$ Robyn Miller $^{3}$. \\ Helen Skouteris ${ }^{1,4}$
}

Accepted: 3 December 2021 / Published online: 11 January 2022

(c) The Author(s), under exclusive licence to Springer Nature Switzerland AG 2021

\begin{abstract}
The aim of this study was to explore and better understand the enablers and barriers of implementation and how these impact on the organisational successes and challenges of adopting The Sanctuary Model, as perceived by residential care staff. Following ethics approval, three semi-structured interviews and six focus groups were conducted with residential care staff between February and July, 2020. Participants identified a number of enablers, presented in the subthemes: (a) social support systems and resources; (b) shared trauma-informed knowledge and understanding; and (c) leadership and champions. These enablers influenced organisational successes in adopting: (a) the Sanctuary Commitments; (b) the S.E.L.F Framework; (c) Reflective Practice and Supervision; and (d) Trauma Theory. A number of barriers hindering implementation were identified. These were reflected in the subthemes: (a) informal practice; (b) lack of practice-based training; (c) poor introduction to young people; and (d) resources. These barriers impacted on organisational challenges faced in residential out-of-home care including: (a) The Sanctuary Model Toolkit and (b) young people's behaviour and engagement. Comparisons from this study and previous findings identified by executive and upper management staff (decision makers) are discussed. Key findings indicate that when implementing, sustaining and embedding The Sanctuary Model, organisations need to become trauma-informed rather than 'do' trauma-informed care and organisations need to "live and breathe" The Sanctuary Model Commitments, be connected and inclusive of one another, use trauma-informed language and feel safe.
\end{abstract}

Keywords Implementation · Evaluation · Out-of-home care · Residential care staff · Trauma-informed care

\section{Introduction}

Trauma-informed care has become increasingly used across community service organisations and child-welfare services, due to a surge of growing awareness of the impacts of trauma,

Helen Skouteris

helen.skouteris@monash.edu

Emma Galvin

emma.galvin@monash.edu

Renee O'Donnell

renee.odonnell@monash.edu

Julie Avery

julie.avery@monash.edu

Heather Morris

heather.morris@monash.edu

Aya Mousa

aya.mousa@monash.edu

Nick Halfpenny

Nick.Halfpenny@mackillop.org.au including neurodevelopment, psychosocial development, physical and mental health (Becker-Blease, 2017; Putnam, 2006). Not surprisingly, trauma-informed, organisationwide models have been developed to support organisations in addressing the complexities of the impact of trauma on the people who experience trauma and the systems and

Robyn Miller

Robyn.Miller@mackillop.org.au

1 Health and Social Care Unit (HSCU), School of Public Health and Preventive Medicine, Monash University, Melbourne, Australia

2 Monash Centre for Health Research and Implementation (MCHRI), School of Public Health and Preventive Medicine, Monash University, Melbourne, Australia

3 MacKillop Family Services, Melbourne, Australia

4 School of Business, Warwick University, Coventry, United Kingdom 
people who serve trauma survivors (Leitch, 2017). However, the ways in which community service organisations and child-welfare services, such as residential out-of-home care (OoHC), translate and implement trauma-informed theory, has not been understood explicitly (Henry \& Perricone, 2018). In Australia, residential OoHC represents the placement of children and young people (referred to as young people from here on) in staffed homes, with approximately $2-4$ other young people per house (Smales et al., 2020). Despite the growing interest in trauma-informed models, there remain significant gaps in understanding how these models are translated and implemented into practice (Bendall et al., 2018).

\section{The Sanctuary Model}

The Sanctuary Model is an organisational change model developed for community service organisations (Bloom, 2010). The Sanctuary Model represents a traumainformed, organisation-wide, evidence-informed approach for creating or changing an organisational culture and is implemented as an overarching framework, which enables the integration of concurrently providing different therapeutic approaches in a coherent trauma-informed methodology (Esaki et al., 2014).

The Sanctuary Model was originally structured across four pillars, however following advocacy in Australia, a fifth pillar of Cultural Safety has been developed and is an important feature of implementation in Australia. The Victorian Aboriginal Community Controlled Health Organisation (VACCHO) describe cultural safety as "being acceptable to difference, having the ability to analyse power imbalances, institutional discrimination, colonisation, and relationships with settlers. Cultural safety is about providing quality health care that fits with the familiar cultural values and norms of the person accessing the service, that may differ from your own and/or the dominant culture" (Victorian Aboriginal Community Controlled Health Organisation, 2014, p. 1). The five pillars of The Sanctuary Model provide the values, tools and shared language (see Table 1) for people in human service organisations to communicate about trauma and its impacts, striving to create an organisational culture of safety and wellbeing across all levels of the organisation (Bloom \& Farragher, 2013). For organisations implementing The Sanctuary Model, all staff across the organisation must commit to, and uphold the Sanctuary Commitments, and the Sanctuary Toolkit should be integrated into the day-to-day operations of the organisation (Bloom, 2012).

\section{Trauma-informed Care and Child-welfare Systems}

Trauma-informed care is a whole-of-systems approach to organisational change, which aims to embed trauma theory into practice across complex and diverse settings including child-welfare, mental health and education services (Bunting et al., 2019). Trauma-informed care became increasingly popular after findings from the Adverse Childhood Experiences (ACE) study demonstrated a relationship between adverse experiences during childhood and poor, life-long health outcomes (Felitti et al., 1998).

Child-welfare systems interact with and serve children and their families who have experienced adversity and trauma, including inter-generational trauma, on a day-today basis and are progressively committing to, or have a desire to, provide trauma-informed care (Bunting et al., 2019). Whilst traumatic experiences are common and affect a large proportion of the child-welfare population, translating trauma-informed care to practice and the consistency of implementation across service settings is challenging (Wall et al., 2016). Service providers and the policies set up in these organisations must respond to the needs of clients with a lived experience of trauma that go beyond a clinical or treatment response, to ensure best outcomes for clients and to support service providers in dealing with the potential for vicarious trauma (Wall et al., 2016). Vicarious trauma is the cumulative, transformative stress that affects those who work with survivors of adverse, traumatic events (Bloom, 2003). Despite the increasing popularity of trauma-informed care models, there is an absence of research exploring the implementation of trauma-informed care in child-welfare services (Galvin et al., 2021a; Hanson \& Lang, 2016).

\section{Understanding the Importance of Implementation}

In order to understand the potential value of a model or framework, we must first understand the process of implementation. Indeed, successful implementation of models and frameworks is critical for achieving intended effects and outcomes, although research indicates that translating the theory of trauma-informed care into child-welfare practices and implementing models and frameworks aimed at improving client outcomes, can be complex (Henry \& Perricone, 2018; Skouteris, 2021).

Model and framework outcomes have been associated with the quality of implementation, hence, understanding the implementation of trauma-informed models can help achieve the intended effects (Durlak, 2011). Comprehending which elements of the model or framework were delivered, how they 
Table 1 The Five Pillars of The Sanctuary Model

\section{Pillar 1. Cultural Safety}

An environment that is safe for people: where there is no assault, challenge or denial of their identity, of who they are and what they need. It is

about shared respect, shared meaning, shared knowledge and experience, of learning, living, and working together with dignity and truly listening.

\section{Pillar 2. Trauma Theory}

A shared understanding of trauma, how traumatic experiences have a direct effect on thoughts, feelings and behaviours, and recognising organisational trauma

\section{Pillar 3. S.E.L.F (Safety, Emotions, Loss, Future) Framework}

A shared language used to understand and solve problems. The framework can be used for client services or treatment, interpersonal and organisational problem solving.

\section{Pillar 4. Seven Sanctuary Commitments}

A set of shared values that guide individuals and organisations away from trauma-reactive behaviours.

\section{Nonviolence}

Counteract the violence and lack of safety that people have often experienced as part of their trauma whether it be physical, psychological, social, moral or cultural.

\section{Emotional Intelligence}

Create an environment in which community members understand the relationship between past experiences and current behaviours and emotions.

\section{Democracy}

Democracy replaces feelings of helplessness by requiring active participation and empowerment in decision making by all community members.

\section{Open Communication}

This commitment creates a community that accepts and encourages the expression of emotions and openly explores interpersonal and organisational issues.

\section{Social Responsibility}

This commitment focuses on building a community in which people feel a sense of responsibility and care for each other, in which people are held accountable for their actions.

\section{Social Learning}

Social Learning promotes collaborative thinking and problem solving. It also mitigates the isolating effects of shame by viewing mistakes as positive learning opportunities.

\section{Growth and Change}

Evaluate current behaviours while focusing on the future by setting achievable goals and breaking dysfunctional patterns.

\section{Pillar 5. Sanctuary Toolkit}

A set of practical interventions that reinforce trauma theory, the Seven Commitments and S.E.L.F.

\section{Community Meetings}

A set of three questions that ask community members to be attuned to their feelings, be future focused (goal for the day) and engage in safe help-seeking and giving.

\section{Red Flag Reviews}

A response to critical incidents that follows a protocol that focuses on solutions rather than problems.

\section{Safety Plans}

A visual reminder of interpersonal emotion management practices including activities, techniques or skills that can be used during triggering situations.

\section{Sanctuary Psychoeducation}

Educational materials and activities about the effects of trauma, techniques for managing these effects and strategies for creating healthy dynamics in groups.

\section{SELF Care Planning}

A framework for creating individualised plans that outline a set of activities one commits to do to manage stress and focuses on cultural, physical, psychological, social and ethical safety and wellbeing

\section{Core Team}

A cross section of staff from all levels of the organisation who ensure the execution of the implementation process by role modelling the values and practices, monitoring implementation steps and addressing organisational challenges.

\section{Supervision}


Table 1 (continued)

Team Meetings

Allow teams to reflect on their practices, discuss team functioning and service delivery issues

SELF for Cultural Healing

A framework to understand the complexity of the barriers facing Aboriginal people and communities in their recovery process.

Adapted from (Esaki et al., 2014) pages 125 - 130 and MacKillop Family Services

were delivered, and what factors impacted on delivery, provides context to the assessment of internal and external validity, and accurately interpreting outcomes (Durlak, 2011). The findings of a systematic review of over 500 studies revealed that effective implementation is associated with better outcomes, particularly if providers modify their interventions to better suit their organisational and/or community environment during implementation, replicating some elements of the program and modifying others (Durlak \& DuPre, 2008). Durlak and DuPre (2008) concluded that identifying the factors that affect the implementation process, and ascertaining which elements of the intervention were delivered successfully through evaluation, can provide organisations with the knowledge and understanding of how an intervention can be embedded effectively to achieve maximum impact.

Although trauma-informed models and frameworks are becoming increasingly available, and organisations and systems have the desire to provide trauma-informed care, there remains a lack of research evaluating: (1) the implementation strategies which enable and hinder implementation in complex organisational systems; and (2) the ways by which these implementation strategies impact the adoption of trauma-informed care models (Ashmore, 2013; Henry \& Perricone, 2018). In order to address these gaps, and better translate theory into practice, it is important to explore the perceived enablers, successes, barriers and challenges of implementation. Exploring and evaluating these aspects, as perceived by staff whilst implementing a trauma-informed model, can provide key observations that will foster the translation of research and theory into practice, and provide astute insights into how models, more generally, can be embedded and sustained in a complex, community-based service.

\section{Study Aims}

The aim of this study was to explore and better understand the enablers and barriers of implementation and how these impact on the organisational successes and challenges of adopting The Sanctuary Model, as perceived by residential OoHC staff. Findings from the current study will inform enhancements to The Sanctuary Model that are likely to foster health and social care outcomes for young people living in residential OOHC.

\section{Methods}

\section{Study Design and Setting}

MacKillop Family Services is a community service organisation providing services across Australia in Victoria, Western Australia, Australian Capital Territory and New South Wales. The Sanctuary Model has been implemented across MacKillop since 2012. Throughout this time, MacKillop have implemented The Sanctuary Model as an overarching framework, while concurrently training carers and staff in a range of evidence-based and evidence-informed models underpinned by strength-based, child-centred, familyfocused practices including: Therapeutic Crisis Intervention, Healing Matters, Eye Movement Desensitisation Reprocessing (EMDR), Therapeutic Life Story Work, Behaviour Support Planning and Power to Kids.

This study was conducted in Victoria, where there are different models of residential care; that is, MacKillop operates therapeutic residential care (additional staffing and access to a therapeutic specialist; total $n=14$ houses in Victoria) and general residential care (total $n=41$ houses in Victoria). This qualitative study involved semi-structured interviews completed in-person and via telephone, and focus groups which were completed online via Zoom, due to COVID-19 restrictions and lockdown. Purposive sampling was used to recruit potential study participants and residential homes within MacKillop Family Services.

\section{Ethics}

This study was approved by the Monash University Human Research Ethics Committee (Project ID: 17520) and the 
MacKillop Family Research Approval Panel. All participants were provided with an explanatory statement and an opportunity to ask questions, after which they provided informed written or verbal consent prior to taking part in the study.

\section{Participants}

For the purpose of this study, participants were recruited from MacKillop Family Services by a MacKillop Liaison and a study investigator (first author EG). The participants in this study included area managers, coordinators, supervisors, case managers, residential care workers, a principal practitioner, therapeutic specialists (clinicians), and an educator. Area Managers are members of the Management Leadership Team and are responsible for overseeing the delivery of day-to-day operations of programs across their jurisdiction. Residential Care Coordinators ensure programs deliver high quality services to vulnerable young people within a trauma-informed framework. The House Supervisor is responsible for overseeing the daily care of young people. Case Managers provide highquality case management and support services. Residential Care Workers participate in the day-to-day operation of the house, which includes responding to the young person's emotional and functional needs, all administrative requirements and household duties. Therapeutic Practitioners are clinicians responsible for guiding residential care workers' interactions with young people, engaging families to support their young person in care, conducting assessments and developing therapeutic treatment plans. Principal Practitioners are unique to MacKillop Family Services, and are senior staff who lead the development and improvement of service delivery practice with a focus on The Sanctuary Model and supervise therapeutic practitioners. Children in Residential Care (CIRC) Educators support residential care workers in encouraging young people to participate in education programs.

Thirty-eight residential care staff across all levels of residential OoHC (Fig. 1) participated in this study. Seven residential houses participated in six focus groups and were located across regional and metropolitan Victoria, Australia (focus group in Regional Victoria included two homes, 1 therapeutic and 1 general residential home as these homes conduct team meetings together). All participants had completed the three-day Sanctuary Model training program, at the time it was introduced or as part of their induction at MacKillop Family Services. A summary of participant demographic information has been provided in Table 2.

\section{Procedures}

\section{Data Collection}

Three semi-structured interviews with an Area Manager, House Supervisor and Residential Care Co-ordinator were conducted by the first author (EG). Six focus groups were facilitated by the first author during team meetings with
Fig. 1 MacKillop Family Services Residential Out-of-Home Care Staff Structure

\section{Staff Structure Victoria}
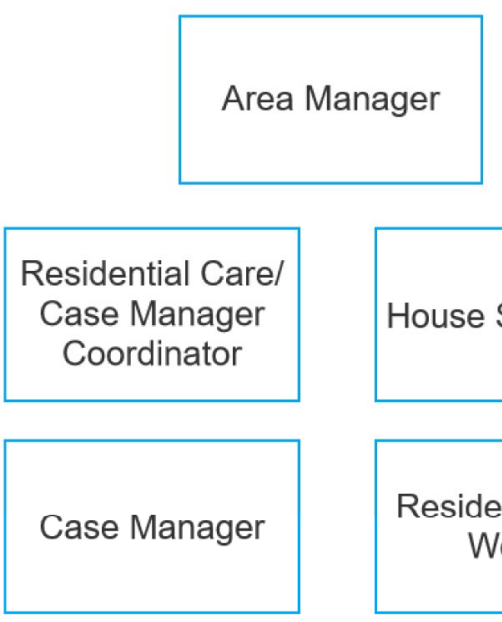

TRC, Therapeutic Residential Care

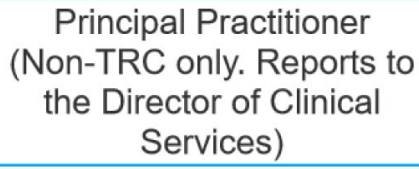

Therapeutic Specialist Practitioner (TRC only. Reports to the Principal Practitioner)

Therapeutic Residential Care Worker (TRC only) 
Table 2 Participant Characteristics

\begin{tabular}{ll}
\hline Participant Characteristics & $\begin{array}{l}\text { Number of } \\
\text { Participants }\end{array}$ \\
\hline Role & 1 \\
Area Managers & 1 \\
Residential Care Coordinators and House Supervisors & 8 \\
Case Managers and Residential Care Workers & 25 \\
Principal Practitioner and Therapeutic Specialists & 3 \\
$\quad$ (clinicians) & 1 \\
Children in Residential Care (CIRC) Educator & \\
Type of Home & 24 \\
General Residential Care Home (n=4 homes) & 14 \\
Therapeutic Residential Care Home (n = 3 homes) & \\
Location of Home & 27 \\
Metropolitan (n=5 homes) & 11 \\
Regional (n=2 homes) & \\
Gender & 25 \\
Female & 13 \\
Male &
\end{tabular}

Residential Care Coordinators, House Supervisors, Case Managers, Residential Care Workers, Principal Practitioners, Therapeutic Specialists and a Residential Care Educator ( $\mathrm{M}=6$ participants, $\mathrm{SD}=2$ participants). Here, the first focus group was co-facilitated by the fourth author (HM). Interviews and focus groups were conducted for approximately one hour $(\mathrm{M}=54.50 \mathrm{~min}, \mathrm{SD}=9.40 \mathrm{~min})$. Interviews were conducted either in person at the residential care home or over the phone and focus groups were conducted online via Zoom, between February and July, 2020. Interviews were conducted for participants who do not or could not attend the team meeting in which the focus groups were conducted.

Open-ended and non-leading questions formulated an interview guide to identify and explore the perceived enablers, successes, barriers and challenges of implementation. Participants were asked to share their experiences of The Sanctuary Model, and how they use the model in their daily practices; for example: (1) Tell me about [tool/ commitment/S.E.L.F/trauma theory], (2) what does that look like in your home?, (3) what has acted as a barrier and/or been difficult to implement?, (4) what impact does that have on young people/your practice?, and (5) what has supported your practice of The Sanctuary Model? The interviewer/facilitator used a combination of techniques during interviews and focus groups (e.g., probing, clarification and confirming responses when required) to encourage further exploration.

\section{Data Variables and Analysis}

Participant roles and location of the homes (regional or metropolitan) were collected via internal records. Gender and type of residential care service (therapeutic or general) were collected prior to the commencement of the interview or focus group.

The interviewer and co-facilitator took field notes during interviews and focus groups, and debriefed immediately after. Data saturation was met after a total of three interviews and six focus group sessions, once it was recognised that no new themes or concepts were being uncovered (Fusch \& Ness, 2015).

Transcripts were entered into NVivo (QSR International Pty Ltd., 2020) prior to coding. Inductive and deductive analysis methods were used with thematic coding. Using deductive coding, outcomes that corresponded to the overarching themes of enablers and successes, barriers and challenges were identified. Inductive coding was then applied to identify emerging sub-themes. Emergent themes were consistently compared and crosschecked within the data. Emerging themes were shared with residential care staff, executive staff and upper management staff at MacKillop Family Services via meetings to facilitate member checking. Two researchers (EG and JA) independently coded the transcripts to increase the rigour of the analysis. Codes, themes and sub-themes were cross-checked for consistency and discrepancies were discussed to ensure research integrity and prevent researcher bias. Consensus was reached on all codes. This study was guided by the Consolidated Criteria for Reporting Qualitative Research (COREQ) checklist (Tong et al., 2007).

\section{Results}

Four key themes relating to the implementation of The Sanctuary Model in residential OoHC are presented: (1) Enablers influencing implementation; (2) Organisational successes of implementation; (3) Barriers influencing implementation; and (4) Organisational challenges of implementation. Within these major themes, 13 subthemes and supporting quotes were identified. For the purpose of this research, we defined enablers and barriers as implementation strategies that facilitated (enablers) or hindered (barriers) implementation of The Sanctuary Model. Organisational successes and challenges are viewed as constructs of The Sanctuary Model perceived to have been effectively (successes) or ineffectively (challenges) implemented in residential OoHC. 


\section{Theme 1: Enablers Influencing Implementation}

It was clear from discussions with residential care workers that there were a number of implementation strategies that influenced their ability to implement The Sanctuary Model with young people in residential OoHC. This is reflected in the subthemes: (a) social support systems and resources; (b) shared trauma-informed knowledge and understanding; (c) leadership and champions.

\section{(a) Social Support Systems and Resources}

The majority of participants defined enablers of implementation as having opportunities to reflect on, support, guide and acknowledge the values and therapeutic approaches of care. These concepts were often associated with social support systems and resources including Care Team Meetings (entire care team meets to discuss the young people), Local Sanctuary Practice Teams (LSPT; Sanctuary leaders and champions in each region meet to provide leadership and support in the implementation of The Sanctuary Model), Team Meetings (staff in the home), House Meetings (staff and young people in the home), and funded positions such as Therapeutic Specialists or Principal Practitioners.

Residential care staff expressed that these systems and resources are "a useful vehicle and mechanism to embed the model", because they provide an opportunity for connection, communication and support. For example:

We come together and work out, how do we implement this in practice, how do we communicate this with the young person...how can we best support this young person and keep them safe (Residential Care Coordinator, Interview 3).

Therapeutic Specialists and Principal Practitioners were identified as vital "on-the-floor supports" who help "encourage staff to think of ways to implement and use Sanctuary". Social support systems and resources were also identified as opportunities to include young people in decision making and to hear their voices:

Our young people also attend the care team meeting, where they're given the opportunity to express their feelings and wishes. So that's some of the elements where we offer young people the chance to get involved and have a say in their care (Case Manager, Focus Group 1).

\section{(b) Shared Trauma-informed Knowledge and Understanding}

Across residential care homes, it was apparent that shared knowledge, language and understanding of trauma-informed care reinforced the implementation of The Sanctuary
Model. Participants communicated that The Sanctuary Model enabled a shared language and understanding of trauma-informed care, providing them with "the framework for knowing what to do and how to intervene". Having a shared language across the organisation facilitated positive changes in the ways in which staff communicated with each other and with young people:

Especially when a young person is distressed or going through an incident or a period of unsettled or uncertain times, it is absolutely that prompt of 'what has happened to you' and a real shift in not just our thinking, but the language that comes out with that as well (Residential Care Coordinator, Interview 3).

Residential care staff expressed that trauma-informed care is the cornerstone of their work and was imperative to working therapeutically with young people. They articulated that shared knowledge and understanding of traumainformed care provided "common ground" and "made most of the homes therapeutic", even if they are not labelled as therapeutic residential care homes. Participants acknowledged that having a "trauma-lens and understanding" has shifted their thought patterns from placing blame to exploring trauma histories and linking these with current behaviours. Through shared knowledge and understanding of trauma, residential care staff recognised a positive shift towards providing "therapeutic, attuned care" to young people and understanding and responding to challenging behaviours:

I think any house could provide very therapeutic, attuned care to the young people in care. It's the understanding, and the knowledge, and the professionalism of the staff working, that make the difference (House Supervisor, Focus Group 2).

\section{(c) Leadership and Champions}

All residential care staff agreed that embedding leaders and champions that guide and support the values of the model has fostered implementation. Participants expressed that having champions provided a "safe supportive layer". Leaders and champions of The Sanctuary Model were described as providing strength and support, offering further opportunities to "reach out, if we're not comfortable going to someone in our direct team". Although they acknowledge a lack of practicebased training, all participants had identified one staff member (a Sanctuary Model trainer) to whom they felt comfortable reaching out to. This staff member was acknowledged as a "leader and champion of Sanctuary", who has delivered 'refresher' workshops, providing an opportunity to "get clarity", "ask questions" and "provide guidance”. 
In terms of The Sanctuary Model having an impact on young people in care, residential care staff agreed that The Sanctuary Model would not "impact on young people at all, if it isn't impacting me and I am really embracing it". For example:

You need to get the staff on board and get the staff doing it in full circles with each other, and inevitably it will trickle down to young people and it also role models it, which is how it is more accessible for young people (Residential Care Worker, Focus Group 6).

\section{Theme 2: Organisational Successes of Implementation}

As a result of the implementation enablers, residential care staff identified four constructs in which The Sanctuary Model had been effectively implemented. These are presented in the subthemes: (a) the Sanctuary Commitments; (b) the S.E.L.F Framework; (c) Reflective Practice and Supervision; and (d) Trauma Theory.

\section{(a) Sanctuary Commitments}

The Sanctuary Commitments were described as values that are inherent in "what we do" and "the way we work with each other and young people". It was evident that the social support systems and resources, leaders and champions that enabled implementation, had a direct impact on the successful implementation of the Sanctuary Commitments. To illustrate:

Sometimes it is really hard, but there's things in place to reassure staff what their safety is...there's me [House Supervisor], there is management, there's [principal practitioners name]. There is many different levels of debriefing and supervision as well (House Supervisor, Interview 2).

Participants felt that Non-Violence and Safety was an integral part of their work and the commitment of NonViolence by all members of the organisation supports staff when there is "a lot of conflict or instances where staff have been assaulted or fights become physical". Shared traumainformed knowledge and understanding also assisted residential care staff with educating young people "on safety, and what safe is and what safe isn't".

Emotional Intelligence and Open Communication were identified as key components on setting the culture within the homes. Participants acknowledged that being emotionally intelligent "helps you unpack crises or incidents" and "the reason behind the behaviour". They also discussed how young people "are in-tune with us and they pick up things far sooner than we do", indicating that staff need to be attuned to their own emotions and "openly communicate" with their social support systems and resources and young people in their care. For instance:

If a young person is having a moment, an outburst, an incident, or presents with a concerning behaviour, I think it can be difficult for some carers to unpack the reason behind the behaviour if they aren't emotionally intelligent (Principal Practitioner, Focus group 2).

\section{(b) Safety, Emotion, Loss and Future (S.E.L.F) Framework}

When reflecting on what has been implemented with fidelity, residential care staff identified the S.E.L.F framework (see Table 1), noting that this framework provided the tools and concepts to have open and honest discussions with young people and "support them, to help them regulate, and support themselves emotionally". Social support systems and resources, leaders and champions provide a safe space to "sit down with them and unpack things", whilst the framework helps staff approach conversations with a "vision" to "create change together" and "respond appropriately to their [young people's] needs". Participants also acknowledged the importance of using the S.E.L.F framework within the social support systems and resources to explore "significant incidences". For example:

I think that it's really, really important that when the incidences happen, it's spoken about, that people explore it, that you have support for the whole team to get out of potentially negative spaces and get them just looking forward and getting different perspectives (House Supervisor, Focus Group 6).

\section{(c) Reflective Practice and Supervision}

Reflective Practice is unique to MacKillop and is a method of supervision aimed to enhance practice through the delivery of appropriate, safe and regular reflection opportunities, learning from experience and reflecting on practice, in which all team members associated with the house are expected to attend. Here, principal practitioners (general residential care) and therapeutic specialists (therapeutic residential care) guide reflective practice.

Reflective practice was recognised as a tool that encouraged staff to reflect on their practice in the residential care house and draw connections with The Sanctuary Model. Reflective Practice provided staff an opportunity to use the shared trauma lens that The Sanctuary Model provides, and to reinterpret the behaviours displayed by the young people. Participants described Reflective Practice as providing a "safe environment", where they feel "supported" in "having 
honest conversations" and "debriefing from situations", without being "judged or criticised". For instance:

Reflective Practice...it helps us move, change our practice, adapt, perspective...it's also important to just be together, and process some of the things that have happened (Residential Care Worker, Focus Group 3).

In addition to Reflective Practice, residential care staff identified Supervision (see Table 1) as a tool that enabled staff to reinterpret incidents in the home by further connecting trauma theory. Supervision provided supportive opportunities to "reflect back on" incidents and "acknowledge" how The Sanctuary Model was/wasn't used in practice. Participants expressed that Supervision provided an opportunity to not only connect trauma theory with practice, but also provided an opportunity to explore trauma theory. To illustrate:

I will then hold the team accountable in terms of their practice around how they implement it, the way they work with kids, and if it reflects Sanctuary (House Supervisor, Interview 2).

\section{(d) Trauma Theory}

The Trauma Theory (see Table 1) embedded in The Sanctuary Model was described as an organisational success due to the deliberate focus on not only the young people in care, but also on staff safety and wellbeing. With a conscious focus on staff safety and wellbeing, trauma theory facilitated a shift in the way staff thought about their work practice, articulated by many participants as "what has happened to you, rather than, what is wrong with you". Shared trauma-informed knowledge and understanding encouraged residential care staff to "see the young person behind the behaviour" and they acknowledged that carers "are much better skilled before they go out onto the floor with the young people" as a result.

Participants discussed how trauma theory gave the organisation the "licence" to focus on staff safety and wellbeing and that this focus on safety and wellbeing meant that "even without the name of therapeutic care home, I think any house could provide very therapeutic, attuned care". Most residential care staff conveyed that trauma theory is a "significant part of your role to be able to support a young person" and that having leaders with a shared knowledge of trauma and its effects, enabled staff to "deal with their own trauma" so that they can be more "empathetic" and "very understanding" towards young people in care. For example:

It's not actually just about the outcomes for the kids it's about outcomes for our staff. Staff who are feeling supported and protected and safe at work... and I think then as a result, that then trickles down to the direct care work with our young people (Area Manager, Interview 1).

\section{Theme 3: Barriers Influencing Implementation}

Across residential OoHC, it was evident from discussions with residential care staff that there were a number of implementation strategies that impacted on their ability to implement The Sanctuary Model. This is reflected in the subthemes: (a) informal practice; (b) lack of practice-based training; (c) poor introduction to young people; and (d) resources.

\section{(a) Informal Practice}

When exploring the implementation of The Sanctuary Model in residential OoHC, all homes expressed that the model was implemented "informally". In exploring this further, it was apparent that some tools such as Red Flag Meetings and Community Meetings occur in the homes with young people, however staff do not use the correct "language", "templates" or "processes". It became evident that residential care staff struggle to find the balance of implementing the tools in a "child-friendly", "natural and organic" manner and using the "appropriate language" and "processes", whilst also trying to engage the young people "without them realising". One participant expressed that "The Sanctuary Model is imbibed in the practice, but not explicit". Two participants discussed how informal practices of The Sanctuary Model impact on young people in care:

I feel like we do talk about it, but we don't put a name to it. So, we do talk with young people, we do use Sanctuary Model, but we don't say, this is The Sanctuary Model (House Supervisor, Focus Group 6).

We can introduce young people and their families to The Sanctuary Model until the cows come home but if we're not practicing that within our houses and it's not something that the kids can see we are doing, then it's pointless (Residential Care Coordinator, Interview 3).

\section{(b) Lack of Practice-based Training}

Here, we refer to practice-based training as training opportunities that incorporate real-world examples of implementation strategies to improve interactions and work practices between staff and clients when implementing The Sanctuary Model. Practice-based training integrates the organisational learnings by individuals, groups and communities and offers collective knowledge grounded by 'real-world' practice and experiences.

Residential care staff identified that implementing The Sanctuary Model in the homes was hindered by a lack of 
practice-based training. The Sanctuary Training provided to all staff was described as being a "heavy load of information" and "theory-based", and participants admitted they "left not sure how I would implement it". Most participants expressed being able to "understand it in theory, but can't explain it in practice". More real-world, practical examples would be welcomed and well received by participants.

When reflecting on training opportunities, there was consensus that the initial training "lacks context" to the residential care setting. Residential care staff expressed that they felt they were "winging" implementation and more "structure" and "practical examples" of what The Sanctuary Model "realistically" looks like, and the "challenges you're going to face", will improve uptake of the model in homes. One residential care worker discussed how they would like to see training delivered in the future:

I want it to be focused on workers and what it really looks like, realistically...I think a discussion around how it actually looks in real life in the house, and what other houses are doing to implement it, a bit of a brainstorming (Residential Care Worker, Focus Group 3).

\section{(c) Poor Introduction to Young People}

Residential care staff reflected on the barriers of implementing The Sanctuary Model with young people in their care, and identified a lack of "formal" and "structured" introductions for young people and their families. Some participants identified that "Sanctuary is on the introduction agenda" template when young people enter a home, however acknowledged that this was not "used" or "understood" widely. All residential homes participating suggested that introducing young people to The Sanctuary Model needed to be more "thorough" and less "ad-hoc" in nature.

Participants expressed that young people need to be formally introduced to The Sanctuary Model as it is "how we run the house" and the commitments provide "expectations". There was a general consensus that young people should be provided with a "child-friendly", "picture-based" information booklet, that they can then "look through at their own leisure". To demonstrate:

It would be good to have a more formal and constructed process to introducing it to our young people, because we just make it up as we go. There's no formal, child friendly way of introducing it...Some kids might get a really in-depth, thorough, introduction, where others might get a little bit of information now, a little bit later (House Supervisor, Focus Group 4).

\section{(d) Resources}

When further discussing implementation barriers, participants identified time constraints and limited access to information and resources as major barriers. Poor access to information and resources was noted as a significant barrier to implementation of the Sanctuary Model, as the majority of resources are only available on the intranet (internal site for MacKillop). This presented as a major barrier as the "intranet is only available when you're in the house" and residential care workers admitted they "can't access the intranet when you're offsite".

However, staff also acknowledged that they "don't have the time to be able to sit on a computer", "clicking away at the button", trying to find resources when "there is a lot happening in the house and you have young people calling your name and other tasks to do". The demands of working in residential care, together with the "high complex clinical needs of our young people", has left staff with a "dearth of capacity and time". Participants admitted they "omit community meetings" due to a "short timeframe to do a handover" and that carers have "to make choice points along the way", therefore often prioritising the needs of the young people over implementing The Sanctuary Model, rather than linking them together. To illustrate:

When we're speaking of what some of the constraints may be...just understanding that we're managing a very extreme level of complexity, which makes it hard to think we're going to do the SELF model today (House Supervisor, Focus Group 5).

\section{Theme 4: Organisational challenges of implementation}

As a result of the implementation barriers, residential care staff identified a number of constructs in which The Sanctuary Model had been ineffectively implemented within residential OoHC. These are reflected in the subthemes: (a) the Sanctuary Toolkit; and (b) young people's behaviour and engagement.

\section{(a) The Sanctuary Toolkit}

Residential care staff wrestled with implementing Psychoeducation, Safety Plans, Community Meetings, and Red Flag Meetings (see Table 1). The informal practice of The Sanctuary Model and lack of practice-based training for residential care staff contributed to participants articulating the challenges of implementing Psychoeducation. Residential Care Workers and House Supervisors expressed their belief 
that Psychoeducation is too "clinical" and "educational" and admitted that they typically "leave it to the Therapeutic Practitioners". For example:

I think the roles get blurred for carers, and particularly with young people, because we sit there and say, 'hang on, I'm just a carer, you need to talk with your clinical team, your aod workers, your counsellors (Residential Care Worker, Focus Group 4).

Residential Care Workers and House Supervisors felt that wearing their Safety Plans around their necks was a major safety concern within the residential home, especially around some young people who like to "grab at the keys or lanyard", and thus were rarely implemented. Most Residential Care Workers admitted that they do not wear their Safety Plans as they are not child-friendly, and as a result, forget about them. Participants also admitted that they discussed Safety Plans on occasion with young people, however they rarely completed a formal template.

The implementation of Community Meetings with young people was described as "difficult", in terms of engaging and encouraging young people to "broaden it out [responses to the three Community Meeting questions] beyond, 'good', 'have dinner"'. Residential Care Workers and House Supervisors felt that Community Meetings were "too formal" for young people, however acknowledged the creativity and flexibility of being able to adapt the questions when trying to engage young people in a Community Meeting. Residential Care Workers and House Supervisors also admitted that they neglect to have Community Meetings at staff change-over, and attributed this to being "time poor", and believing that they can "read the room, or, read a person before they've even entered the room". Some participants felt that some responses are a "latter of the truth" as they had an impression that their feelings may not always be appropriate to share in a work environment. To demonstrate:

I probably wouldn't be the only one in this room that has been feeling like absolute $\mathrm{s}^{* * *}$, screaming to the top of your lungs, but you're not going to tell anyone that, you're going to say I'm feeling a little jaded today, you're not actually going to say I really shouldn't be here right now, I feel terrible (Residential Care Worker, Focus Group 1).

Whilst exploring the perceived organisational challenges, it became clear that residential care staff struggled with Red Flag Meetings. Common perceptions of Red Flag Meetings were that when they are utilised, they are "misused", "nonproductive" or a "token gesture". Residential care staff felt as though there is an "unwritten rule that if you call a Red Flag Meeting, it is going to be frowned upon [by senior staff]" and that "people feel they don't have a licence to be able to use Red Flags as they were intended". Informal practice hindered the implementation of Red Flag Meetings in residential care as participants acknowledged that they are rarely called. However, multiple participants identified that they call 'urgent' meetings frequently and expressed that these meetings encompass the meaning of a Red Flag Meeting without actually using the correct language or processes:

"We could see that potentially we were having a Red Flag Meeting just not really calling it a Red Flag Meeting" (Therapeutic Specialist, Focus Group 1).

I think that the language that we are using around those meetings, we're not calling them Red Flag Meetings. I think that when we're not using the appropriate language for these things and we're not calling them what we should be calling them, then really, they're not Red Flags (Residential Care Coordinator, Interview 3).

\section{(b) Young people's behavior and engagement}

In addition to implementing The Sanctuary Toolkit, residential care staff identified that young people's behaviour and engagement within the home was a cumulative challenge of implementing The Sanctuary Model. Focusing on the young person and what is happening around the home each day is a priority for Residential Care Workers and House Supervisors. Participants felt as though the young people's behaviour, attitude, complex clinical needs and engagement can shape the dynamics of the home and impact on how they implement The Sanctuary Model on a day-to-day basis.

Residential care staff discussed the complexities of implementing The Sanctuary Model with young people, particularly around engaging them. Participants explained that each young person's "behaviours and challenges are different", and that it is a constant struggle "thinking of things to get them engaged" or "engaging them without them realising".

Young people absent or missing from placement were described as being particularly hard to engage, and that "you can't implement it with kids who are not here". Alongside young people absent or missing from placement were the complex clinical needs of the young people and the pushback they receive from young people when trying to implement The Sanctuary Model. Residential care staff felt that implementation had to be "as natural as possible" as "forcing them to sit around a table, at this specific time" will cause them to disengage and push-back, with one home admitting that young people see it is as a "chore" and "expect something in return" if implementation is "forced". For example:

With a lot of the young people, I think it can be a bit more challenging sort of being like, 'alright, let's sit down and use these steps', and, you might just have young people telling you to 'eat a sock', or to 'suck on that' (Residential Care Worker, Focus Group 5). 


\section{Discussion}

The aim of this study was to explore and better understand the enablers and barriers of implementation and how these impact on the organisational successes and challenges of adopting The Sanctuary Model, as perceived by residential care staff. Through engaging with residential care staff at MacKillop Family Services, we have gained insights into the perceived enablers, successes, barriers and challenges experienced on the forefront of the organisation and can compare these experiences to those working in back-ofhouse services. Key findings in this study were similar to those found with both executive and upper-management decision makers and will be discussed below (Galvin et al., 2021b).

\section{Enablers and Organisational Successes}

Surprisingly, the pivotal implementation strategies that enabled implementation identified by residential care workers were the same as those identified by decision makers, as previously reported (Galvin et al., 2021b). These included social support systems, shared trauma-informed knowledge and understanding, and leadership and champions. Decision makers did not identify Therapeutic Specialists or Principal Practitioners when discussing enablers of implementation in residential OoHC, suggesting that decision makers are not as aware of the roles as they need to be, which may impact on them building the culture within the homes (Galvin et al., 2021b).

It is evident that having a shared level of understanding and knowledge of trauma-informed care is vital to staff engagement across all levels of the organisation and there was general consensus that this shared knowledge and understanding provided a deeper understanding into the trauma a young person may have experienced. Previous research suggests that the acquisition of trauma-informed knowledge and understanding has the ability to shift attitudes about working in a trauma-informed setting and the association between attitudes and shifts in behaviour when interacting with traumatised clients has been supported (Brown, Baker, \& Wilcox, 2012). Our results show that through shared knowledge and understanding, there is a greater opportunity to include all staff in championing, role modelling and encouraging the implementation of The Sanctuary Model effectively, which in turn, creates an organisational culture of staff safety and wellbeing.

Our results indicate that social support systems and resources supported the implementation of reflective practice and supervision, which aligns with previous research findings suggesting that organisations implementing trauma-informed approaches need to embrace collaborative approaches incorporating reflection and systemic thinking (McNamara, 2010). Present findings also indicated that having leaders and champions who support staff in processing the impacts of vicarious trauma and the negative impacts of constant exposure to details of childhood trauma have encouraged a trauma-responsive, organisational culture.

The organisational successes were also similar across all levels of the organisation, strongly associating the enablers of implementation with the effective and successful use of the Sanctuary Commitments, S.E.L.F framework, and Reflective Practice (Galvin et al., 2021b). In addition, residential care workers identified Supervision as a tool used successfully to discuss, plan, share and acknowledge how staff are implementing The Sanctuary Model; and, with a specific focus on staff safety and wellbeing, Trauma Theory was identified as the backbone of direct care with young people in residential care. The need for adequate supervision, where staff are provided a safe space to debrief has also emerged in previous research (Brend \& Sprang, 2020; Eastwood \& Ecklund, 2008).

The enablers and organisational successes of implementing The Sanctuary Model in residential care and across executive and upper management, indicate multiple layers of support for staff, that all contribute to the culture of the organisation. Staff are encouraged to look beyond the behaviours of young people in organisations that implement trauma-informed care, and by shifting their knowledge around trauma and its effects, staff can provide improved care for the young people, providing them with greater opportunities for improved health and wellbeing (Center for Substance Abuse Treatment (US), 2014; Hodgdon et al., 2013; Wall et al., 2016). It is evident that to successfully implement The Sanctuary Model, organisations must not only 'do' trauma-informed care, they must 'be' traumainformed. Staff must live and breathe The Sanctuary Model Commitments, be connected and inclusive of one another, use trauma-informed language and feel safe for The Sanctuary Model to be implemented and practiced well.

\section{Barriers and Organisational Challenges}

Whilst staff acknowledge that they need to be traumainformed to support the young people in their care and facilitate the implementation of The Sanctuary Model, they also identified numerous barriers and organisational challenges when implementing the model. Residential care staff in this study and executive and upper management staff in our previous study identified dissimilar barriers and organisational challenges to implementation (Galvin et al., 2021b). This is concerning as previous research shows that executive 
and upper management leaders play a vital role in setting the organisational culture through determining the nature of organisational systems, procedures, structures and contexts (Aarons et al., 2015; Oke et al., 2009). As such, a poor understanding of the barriers and challenges faced by the staff who are directly impacted by management decisions, can detrimentally influence the implementation and sustainability of these decisions (Aarons et al., 2014).

Residential care staff identified the informal implementation of the model as the most significant barrier to implementing The Sanctuary Model, where a lack of appropriate language and staff struggling to find the balance between implementing the model and keeping it organic and natural, as to not disengage the young people influenced informal practice. Some participants also struggled with being able to adapt the model into a child-friendly practice that they were comfortable with delivering. These findings are alarming as previous research have suggested that improved implementation and sustainability occurs when providers can adjust and adapt a model, making it more effective for the community and setting in which it is being implemented (Durlak \& DuPre, 2008; Fixsen et al., 2005; Moore et al., 2013).

Residential care workers also felt that a major barrier for successfully implementing and embedding The Sanctuary Model into residential OoHC was the poor and inconsistent introduction of the model to the young people in their care. After providing these results to MacKillop Family Services, the Beginners Guide to Sanctuary, an introductory resource for young people has been developed. The resource provides child-friendly, inclusive and culturally appropriate information and pictures that residential care staff can use to guide their introductions and young people can keep the resource to look through and ask questions in their own time.

In the current study, residential care workers found that these barriers made it challenging to engage young people with The Sanctuary Model and implement The Sanctuary Toolkit. Participants indicated that the clinical complexities, behaviour and engagement of young people and the stream of continuous incidents, impacted on their time and capacity to effectively implement The Sanctuary Model. These findings reveal that residential care workers require support in connecting the theory of The Sanctuary Model and how to adapt the Sanctuary Toolkit into their day-to-day practices with traumatised young people in residential OoHC. It is evident that the accumulation of poor introductions to young people, lack of real world, practical training and the feelings of limited time and capacity, contribute to the overall challenges faced by residential care staff when implementing models and interventions.

Although staff across all levels of the organisation reported a deeper understanding of trauma theory, when looking at the results as a whole, it is evident that staff are implementing elements of The Sanctuary Model with fidelity, however informally. It is therefore suggested that the barriers and organisational challenges go beyond practicebased training and instead, infer a need to better connect the theory of The Sanctuary Model to their practice. When staff are not using the appropriate language, identifying their practice as part of, or intently linking their work back to, The Sanctuary Model, it is understandable why they may feel that The Sanctuary Model is not reaching their practice. It is evident within the enablers and organisational successes that although The Sanctuary Model is being implemented across the organisation and impacting on practice, staff struggle to connect 'what they know' with 'how they practice' the model.

\section{Limitations and Implications}

This study should be considered in light of its limitations. First, the information shared in this report represents a single community service organisation. While we have no reason to believe that the residential OoHC setting would greatly differ from other organisations offering the same services, it is unknown whether these study findings generalise beyond this organisation. However, the residential care homes involved in this study were from a combination of general residential OoHC and therapeutic (additional staffing and access to a therapeutic specialist) residential homes both of which implement The Sanctuary Model, and importantly, the study revealed commonalities and shared themes across the homes in regards to the enablers, successes, barriers and challenges experienced during implementation. In future, studies should involve a combination of organisations to address this limitation. Secondly, demographic details such as participant age, time in their current role and time at the organisation were not provided. The researcher followed up with staff to provide this information, however was unsuccessful.

Despite these limitations, the study contributes to the knowledge of implementing trauma-informed care in the child-welfare system. The child-welfare system has been calling for evidence-based, trauma-informed practices, yet many interventions have not been evaluated in this complex setting. As evidence-based interventions continue to be implemented throughout child-welfare systems, organisations require support and guidance on the structural changes needed to reinforce successful implementation and sustainment. To facilitate this, further research is needed to expand our understanding of implementation experiences in the child-welfare system. It is clear that successful implementation in residential OoHC cannot rely solely on the frontline, client-facing workers and more attention should be focused on examining the implications of cross-agency practices. This study offers further insight and understanding into the 
perceived enablers, successes, barriers and challenges experienced by residential care workers whilst implementing The Sanctuary Model.

\section{Conclusions}

Overall, this study highlights important themes about the achievements and impediments of implementing a traumainformed model of care in the child-welfare system. Clearly, organisations need to become trauma-informed rather than do trauma-informed care, to sustain and embed The Sanctuary Model. If organisations are able to connect trauma theory to their practice, they can successfully implement and embed The Sanctuary Model. In turn, The Sanctuary Model can contribute to improvements in the organisational culture, which can enhance the cultural, physical and emotional health and wellbeing of young people in residential care, by supporting the staff working with them.

Acknowledgements We would like to thank all the study participants for their valuable time, effort and insight provided in this research project.

Author Contributions EG contributed to the conceptualization and design of the study, collected data, translated and coded the transcripts, analysed the data, interpreted results and wrote the manuscript. JA coded the transcripts, contributed to the interpretation of results and reviewed the manuscript. RO and AM contributed to the design of the study and critically reviewed the manuscript. HM contributed to the development of the interview guide, co-facilitated a focus group, provided field notes and reviewed the manuscript. $\mathrm{NH}$ facilitated inviting potential participants and reviewed the manuscript. RM reviewed the manuscript. HS determined the conceptualization and design of the study, critically revised the manuscript, supervised the study process and is the guarantor for ensuring the integrity and accuracy of this study.

Funding Statement This research received no specific funding from any public, commercial or not-for-profit sectors.

\section{Declarations}

Conflict of Interest On behalf of all authors, the corresponding author states that there is no conflict of interest

\section{References}

Aarons, G. A., Ehrhart, M. G., Farahnak, L. R., \& Hurlburt, M. S. (2015). Leadership and organizational change for implementation (LOCI): a randomized mixed method pilot study of a leadership and organization development intervention for evidence-based practice implementation. Implementation Science, 10(1). https:// doi.org/10.1186/s13012-014-0192-y

Aarons, G. A., Ehrhart, M. G., Farahnak, L. R., \& Sklar, M. (2014). Aligning leadership across systems and organizations to develop a strategic climate for evidence-based practice implementation.
Annual Review of Public Health, 35(1), 255-274. https://doi.org/ 10.1146/annurev-publhealth-032013-182447.

Ashmore, T. (2013). The implementation of trauma informed care in acute mental health inpatient units: A comparative study. (Masters of Public Health), Massey University, Wellington, New Zealand. https://mro.massey.ac.nz/handle/10179/5855

Becker-Blease, K. A. (2017). As the world becomes traumainformed, work to do. Journal of Trauma \& Dissociation, 18(2), 131-138. https://doi.org/10.1080/15299732.2017.1253401

Bendall, S., Phelps, A., Browne, V., Metcalf, O., Cooper, J., Rose, B., Nursey, J., \& Fava, N. (2018). Trauma and young people. Moving toward trauma-informed services and systems. Melbourne: Orygen, The National Centre of Excellence in Youth Mental Health.

Bloom, S. (2003). Caring for the Caregiver: Avoiding and Treating Vicarious Trauma. In A. Giardino (Ed.), Sexual Assault, Victimization Across the Lifespan (pp. 459-470).

Bloom, S. (2010). Sanctuary: An Operating System for Living Organizations. In N. Tehrani (Ed.), Managing Trauma in the Workplace (pp. 235-251). Routledge.

Bloom, S. (2012). The SANCTUARY MODEL. In C. R. Figley (Ed.), Encyclopedia of Trauma (pp 579-582). https://doi.org/10.4135/ 9781452218595

Bloom, S. L., \& Farragher, B. (2013). Restoring sanctuary: A new operating system for trauma-informed systems of care. https://doi. org/10.1093/acprof:oso/9780199796366.001.0001

Brend, D., \& Sprang, G. (2020). Trauma-informed care in child welfare: an imperative for residential childcare workers. International Journal of Child and Adolescent Resilience, 7, 154-165. https:// doi.org/10.7202/1072595ar.

Brown, S. M., Baker, C. N., \& Wilcox, P. (2012). Risking connection trauma training: a pathway toward trauma-informed care in child congregate care settings. Psychological trauma, 4(5), 507-515. https://doi.org/10.1037/a0025269.

Bunting, L., Montgomery, L., Mooney, S., MacDonald, M., Coulter, S., Hayes, D., \& Davidson, G. (2019). Trauma informed child welfare systems-a rapid evidence review. Int J Environ Res Public Health, 16(13). https://doi.org/10.3390/ijerph16132365

Center for Substance Abuse Treatment (US). (2014). Trauma-Informed Care: A Sociocultural Perspective. In Substance Abuse and Mental Health Services Administration (Ed.), Trauma-Informed Care in Behavioral Health Services (pp 3-33). Retrieved from https:// www.ncbi.nlm.nih.gov/books/NBK207195/

Durlak, J. A. (2011). The Importance of Implementation for Research, Practice, and Policy. Retrieved from Child Trends: https://www. childtrends.org/publications/the-importance-of-implementationfor-research-practice-and-policy

Durlak, J. A., \& DuPre, E. P. (2008). Implementation matters: a review of research on the influence of implementation on program outcomes and the factors affecting implementation. Am J Community Psychol, 41(3-4), 327-350. https://doi.org/10.1007/ s10464-008-9165-0

Eastwood, C. D., \& Ecklund, K. (2008). Compassion fatigue risk and self-care practices among residential treatment center childcare workers. Residential Treatment for Children \& Youth, 25(2), 103 122. https://doi.org/10.1080/08865710802309972.

Esaki, N., Benamati, J., Yanosy, S., Middleton, J., Hopson, L., Hummer, V., \& Bloom, S. (2014). The Sanctuary Model: Theoretical Framework. Families in Society, 94(2), 119-134. https://doi.org/10.1606/ 1044-3894.4287

Felitti, V. J., Anda, R. F., Nordenberg, D., Williamson, D. F., Spitz, A. M., Edwards, V., Koss, M. P., \& Marks, J. S. (1998). Relationship of childhood abuse and household dysfunction to many of the leading causes of death in adults. The Adverse Childhood Experiences (ACE) Study. Am J Prev Med, 14(4), 245-258. https://doi. org/10.1016/s0749-3797(98)00017-8 
Fixsen, D. L., Naoom, S., Blase, K., Friedman, R., \& Wallace, F. (2005). Implementation Research: A Synthesis of the Literature. University of South Florida, National Implementation Research Network.

Fusch, P., \& Ness, L. (2015). Are we there yet? data saturation in qualitative research. Qualitative Report, 20(9), 1408-1416.

Galvin, E., Morris, H., Mousa, A., O’Donnell, R., Halfpenny, N., \& Skouteris, H (2021a). Implementation of The Sanctuary Model in residential out-of-home care: Enablers, barriers, successes and challenges. Children and Youth Services Review, 121, 105901. https://doi.org/10.1016/j.childyouth.2020.105901

Galvin, E ., O'Donnell, R., Breman, R., Avery, J., Mousa, A., Halfpenny, N., Skouteris, H. (2021b). Interventions and Practice Models for Improving Health and Psychosocial Outcomes for Children in Residential Out-of-Home Care: Systematic Review Australian Social Work 1-15. https://doi.org/10.1080/0312407X.2020. 1856394

Hanson, R. F., \& Lang, J. (2016). A critical look at trauma-informed care among agencies and systems serving maltreated youth and their families. Child Maltreat, 21(2), 95-100. https://doi.org/10. $1177 / 1077559516635274$.

Henry, J., \& Perricone, A. (2018). The tale of two counties united by their pursuit of the best interest of children through traumainformed practice. In V. C. Strand \& G. Sprang (Eds.), Trauma Responsive Child Welfare Systems (pp. 231-244). Springer International Publishing. https://doi.org/10.1007/978-3-319-64602-2_14

Hodgdon, H. B., Kinniburgh, K., Gabowitz, D., Blaustein, M. E., \& Spinazzola, J. (2013). Development and implementation of trauma-informed programming in youth residential treatment centers using the ARC framework. Journal of Family Violence, 28(7), 679-692. https://doi.org/10.1007/s10896-013-9531-z.

Leitch, L. (2017). Action steps using ACEs and trauma-informed care: a resilience model. Health \& Justice, 5(1), 5. https://doi.org/10. 1186/s40352-017-0050-5

McNamara, P. M. (2010). Staff spport and supervision in residential youth justice: an Australian model. Residential Treatment for Children \& Youth, 27(3), 214-240. https://doi.org/10.1080/0886571X.2010. 501630.
Moore, J. E., Bumbarger, B. K., \& Cooper, B. R. (2013). Examining adaptations of evidence-based programs in natural contexts. The Journal of Primary Prevention, 34(3), 147-161. https://doi.org/ 10.1007/s10935-013-0303-6.

Oke, A., Munshi, N., \& Walumbwa, F. (2009). The influence of leadership on innovation processes and activities. Organizational Dynamics - ORGAN DYN, 38, 64-72. https://doi.org/10.1016/j. orgdyn.2008.10.005.

Putnam, F. W. (2006). The impact of trauma on child development. Juvenile and Family Court Journal, 57(1), 1-11. https://doi.org/ 10.1111/j.1755-6988.2006.tb00110.x

QSR International Pty Ltd. (2020). NVivo (released in March 2020). Retrieved from https://www.qsrinternational.com/nvivoqualitative-data-analysis-software/home.

Skouteris, H. (2021). Addressing health and social care during and beyond COVID-19: the importance of implementation science. Public Health Research \& Practice, 31(1):e3112103 https://www. phrp.com.au/issues/march-2021-volume-31-issue-1/covid-19-theimportance-of-implementation-science/

Smales, M., Savaglio, M., Morris, H., Bruce, L., Skouteris, H., \& Green, R. (2020). "Surviving not thriving": experiences of health among young people with a lived experience in out-of-home care. International Journal of Adolescence and Youth, 25(1), 809-823. https://doi.org/10.1080/02673843.2020.1752269

Tong, A., Sainsbury, P., \& Craig, J. (2007). Consolidated criteria for reporting qualitative research (COREQ): a 32-item checklist for interviews and focus groups. International Journal for Quality in Health Care, 19(6), 349-357. https://doi.org/10.1093/intqhe/ mzm042

Victorian Aboriginal Community Controlled Health Organisation. (2014). An Introduction to Cultural Safety in Aboriginal Health.

Wall, L., Higgins, D., \& Hunter, C. (2016). Trauma-informed care in child/ family welfare services. 37. https://aifs.gov.au/cfca/publications/ trauma-informed-care-child-family-welfare-services

Publisher's Note Springer Nature remains neutral with regard to jurisdictional claims in published maps and institutional affiliations. 